INPLASY

PROTOCOL

To cite: Li et al. The effect of bicyclol on blood biomarkers of NAFLD: a systematic review and meta-analysis. Inplasy protocol 202080017. doi: 10.37766/inplasy2020.8.0017

Received: 05 August 2020

Published: 05 August 2020

Corresponding author: Zong-Gen Peng

pumcpzg@126.com

Author Affiliation: Institute of Medicinal Biotechnology, Chinese Academy of Medical Sciences and Peking Union Medical College

Support: 2019-I2M-1-001, 81621064.

Review Stage at time of this submission: Preliminary searches.

Conflicts of interest:

None.

\section{The effect of bicyclol on blood biomarkers of NAFLD: a systematic review and meta-analysis}

Li, $\mathrm{H}^{1}$; Liu, NN2; Peng, ZG³.

Review question / Objective: Is bicyclol effective for the improvement of blood biomarkers in NAFLD patients?

Condition being studied: Nonalcoholic fatty liver disease (NAFLD) is a global epidemic without admitted therapeutic agents in clinic. This meta-analysis aimed to assess the efficacy of the marketed hepatoprotective and antiinflammatory drug bicyclol for the improvement of blood biomarkers in NAFLD patients.

Information sources: Pubmed, Embase, Cochrane Library and Chinese databases, including China National Knowledge Infrastructure database (CNKI), VIP-Chinese scientific and technological journal database, Wanfang digital periodical full-text database.

INPLASY registration number: This protocol was registered with the International Platform of Registered Systematic Review and Meta-Analysis Protocols (INPLASY) on 05 August 2020 and was last updated on 05 August 2020 (registration number INPLASY202080017).

\section{INTRODUCTION}

Review question / Objective: Is bicyclol effective for the improvement of blood biomarkers in NAFLD patients?

Condition being studied: Nonalcoholic fatty liver disease (NAFLD) is a global epidemic without admitted therapeutic agents in clinic. This meta-analysis aimed to assess the efficacy of the marketed hepatoprotective and anti-inflammatory drug bicyclol for the improvement of blood biomarkers in NAFLD patients.

\section{METHODS}

Participant or population: Male and female patients were diagnosed as NAFLD with or 
without type 2 diabetes mellitus according to their corresponding guidelines.

Intervention: Bicyclol monotherapy group or bicyclol combined with other medical treatment.

Comparator: Llifestyle intervention (LSI) or other drug monotherapy group.

Study designs to be included: All study designs are acceptable.

Eligibility criteria: Inclusion criteria fulfilled the following criteria: 1) being randomized controlled trial (RCT); 2) male and female patients were diagnosed as NAFLD with or without type 2 diabetes mellitus according to their corresponding guidelines; 3) the average baseline alanine transaminase (ALT) level should be more than $90 \mathrm{U} / \mathrm{L}$ (2 3 times the upper limit of normal values17), while triglyceride (TG) level should be between 2.5 and $5 \mathrm{mmol} / \mathrm{L}$; 4) being published English or Chinese articles. Exclusion criteria were 1) non-clinical studies, non-randomized controlled trials; 2) drug-, viral-, alcohol-, autoimmune-, primary biliary cholangitis, liver decompensation-, or malignancy- or genetic- caused liver injury; 3) studies enrolling fewer than 20 subjects in each group, or treatment time of the studies less than 4 weeks; 4) studies without enough experimental data, such as case reports, reviews, conference abstracts, or biochemical indicator missing.

Information sources: Pubmed, Embase, Cochrane Library and Chinese databases, including China National Knowledge Infrastructure database (CNKI), VIPChinese scientific and technological journal database, Wanfang digital periodical full-text database.

Main outcome(s): Twelve RCTs involving 1008 patients were included. The efficacy of reducing serum $A S T$, TBIL and TC in NAFLD patients by bicyclol treatment was significantly higher than in control group. When the substantial heterogeneity exists, subgroup analyses showed that bicyclol monotherapy significantly decreased the
ALT level and was likely to decrease TG level. Correspondingly, bicyclol combination usage significantly decreased TG level and presented the tendency to decrease ALT.

Quality assessment / Risk of bias analysis: The quality of randomized controlled trials, which assigned as 'high risk', 'low risk' or 'unclear risk' to each item, was assessed independently by two reviewers according to the Cochrane risk of bias tool.

Strategy of data synthesis: The outcome indicators from all included articles were extracted from selected studies and checked by two authors to guarantee the accuracy of data. Review Manager 5.3 was used to analyze the data. Any discrepancies were resolved through discussion.

Subgroup analysis: When the significant heterogeneity exists $(I 2 \geq 75 \%$ and $P<$ 0.10 ), subgroup analysis was conducted according to bicyclol monotherapy and combination usage, and if $I 2$ still over $75 \%$ in the subgroup, descriptive results were provided.

Sensibility analysis: Sensitivity analysis will be conducted through omitting included studies one by one to reflect the impact of individual data on the overall results.

\section{Country(ies) involved: China.}

Keywords: nonalcoholic fatty liver disease; meta-analysis; clinical practice; bicyclol; blood biomarkers.

Contributions of each author:

Author 1 - Hu Li - Conceptualization, analysis, writing original draft, visualization. Author 2 - Nan-nan Liu - Validation of data and analysis.

Author 3 - Zong-gen Peng - Supervision, validation and writing draft. 